\title{
L'inventaire par évaluation visuelle de grosseurs d'arbres, une alternative pertinente aux inventaires forestiers complets
}

\author{
Philippe LeJEunE*, Jacques HÉBERT, Emmanuelle Bousson, Vincent VERRUE, Jacques RondeuX \\ Faculté universitaire des Sciences agronomiques de Gembloux, Unité de gestion des ressources forestières et des milieux naturels, \\ Passage des Déportés 2, 5030 Gembloux, Belgique
}

(Reçu 5 juillet 2004 ; accepté 22 décembre 2004)

\begin{abstract}
Résumé - L'inventaire par évaluation visuelle de grosseurs d'arbres utilise un dispositif d'échantillonnage systématique à taux de sondage élevé (25 à $40 \%)$. Il est proposé comme alternative aux inventaires complets pour la caractérisation de peuplements feuillus mélangés à structure inéquienne. Les arbres contenus dans des unités d'échantillonnage circulaires de 10 ares sont classés «à l'œil » au sein de 5 catégories de grosseur de grande amplitude. Deux modalités de cette méthode, faisant intervenir 1 ou 2 opérateurs (IEVG1 ou IEVG2), sont comparées aux méthodes d'inventaire complet (IC) et d'inventaire par échantillonnage avec mesures des grosseurs (IEMG), dans le cadre d'une expérimentation menée en taillis sous futaie. Aucune différence significative n'est mise en évidence entre IEVG1, IEVG2 et IC pour l'estimation globale du nombre de bois et de la surface terrière, ainsi que pour la distribution du nombre de tiges par classes de grosseur. Par contre, la comparaison avec IEMG montre une sous-estimation légère $(5 \%)$ mais significative, du nombre de bois par hectare dans le cas de IEVG1. Cette sous-estimation apparaît principalement dans les unités d'échantillonnage comportant un nombre élevé de petits bois. Cette méthode d'inventaire peut néanmoins être recommandée pour la description de peuplements feuillus mélangés à structure inéquienne, notamment dans le cadre de la phase d'analyse des aménagements forestiers. Elle s'avère d'autant plus intéressante que des données complémentaires (régénération naturelle, état sanitaire, ...) peuvent être collectées lors du même inventaire et qu'une présentation cartographique des résultats est réalisable à l'échelle du peuplement.
\end{abstract}

inventaire forestier / évaluation visuelle / échantillonnage / aménagement forestier

\begin{abstract}
Inventory with visual evaluation of tree girth: a relevant alternative to complete forest inventories. The inventory technique using visual evaluation is based on a systematic sampling method with a high survey rate (25 to $40 \%)$. It is proposed as a promising alternative to complete inventories for the characterisation of mixed broadleaved uneven-aged stands. Trees located within 0.1 ha sampling plots are visually graded into large size categories. Two modalities of this inventory method, using one or two operators (IGVE1 or IGVE2), have been compared with two other methods: the classical complete inventory (CI) and a systematic sampling inventory with girth measurement (SIGM). The calculations are based upon experimentations carried out in a coppice with standards. No significant difference has been detected between IGVE1, IGVE2 and the complete inventory, concerning number of trees and basal area estimations, considered globally or by girth classes. However, IGVE1 compared with SIGM, provides a slight, but significant, underestimation (5\%) of the number of trees. This is mainly observed in the sampling units comprising a high number of small trees. Nevertheless, inventory method with girth visual evaluation can be considered as a very useful tool for the description of unevenaged mixed broadleaved stands, namely during the stage of analysis of management plans. Furthermore other information useful for the preliminary phase of a forest management (description of regeneration, health status of trees, ...) can also be collected during the same inventory, and a thematic mapping of the results is possible at the stand level.
\end{abstract}

forest inventory / visual evaluation / sampling / forest management

\section{INTRODUCTION}

L'inventaire forestier complet fait partie de l'arsenal de méthodes utilisées en matière d'analyse et d'aide à la décision pour l'aménagement de forêts soumises à une sylviculture intensive. Si cette méthode fournit, a priori, les résultats les plus précis, ils ne sont cependant pas dénués d'erreurs comme le soulignent de nombreux auteurs. Duplat et Perrotte [6] estiment, sans toutefois pouvoir le vérifier, que $95 \%$ des résultats d'un inventaire complet exprimés en surface terrière, sur une étendue de l'ordre de 20 ha, se situent dans un intervalle de
$-15 \%$ à $+10 \%$ autour de la vraie valeur. En outre, un inventaire complet requiert une main-d'œuvre importante et procure des résultats dendrométriques tels que le nombre de tiges, la surface terrière, le volume ou encore divers accroissements, sans donner d'information sur la variabilité spatiale de ces données, ni permettre la récolte d'informations complémentaires relatives à la régénération, par exemple [7]. Dans le contexte d'un aménagement forestier, la récolte des seules données dendrométriques est nécessaire, mais ne suffit pas. C'est précisément sur ce plan que l'inventaire complet montre ses limites et que des méthodes telles que celle par évaluation visuelle constituent

* Auteur pour correspondance : lejeune.p@ fsagx.ac.be 
Tableau I. Récapitulatif des types et des modalités d'inventaires testés.

\begin{tabular}{lccc}
\hline & Nombre d'opérateurs & Nombre de répétitions & Nombre d'UE \\
\hline Inventaire complet & 4 & 1 & - \\
Inventaire par évaluation visuelle & 2 & 2 & 63 \\
- Première modalité & 1 & 1 & 63 \\
- Seconde modalité & 2 & 1 & 30 \\
Inventaire par échantillonnage avec mesures des grosseurs & & \\
\hline
\end{tabular}

une alternative qui peut s'avérer pertinente, principalement pour l'étude des peuplements mélangés d'âges multiples où des données complémentaires telles qu'une description de la régénération s'avèrent importantes pour le gestionnaire forestier.

L'inventaire par évaluation visuelle est inspiré des méthodes d'inventaires typologiques [1] et relève dans ses fondements mêmes d'une méthode par échantillonnage systématique, à taux de sondage très élevé (pouvant aller jusqu'à $40 \%$ ), caractérisée par une simplification des modalités de prise de données de manière à compenser l'effort de mesure supplémentaire imposé par ce taux de sondage. L'évaluation visuelle porte sur le nombre de tiges par essence et par classe de grosseur de large amplitude. Elle remplace les mesures de circonférence de chaque arbre de l'unité d'échantillonnage et permet ainsi de conserver des temps d'exécution réalistes malgré le nombre élevé d' $\mathrm{UE}^{1}$ à parcourir [3].

Cependant, compte tenu de cette simplification et de l'importance du rôle des opérateurs dont la qualité des estimations détermine largement la fiabilité des résultats, il s'est avéré nécessaire de comparer ce type d'inventaire d'une part avec les inventaires complets traditionnellement utilisés dans des forêts feuillues irrégulières en Région wallonne et d'autre part avec un inventaire par échantillonnage incluant des mesures de grosseur précises (inventaire dendrométrique).

Les comparaisons ont été effectuées au sein d'un taillis sous futaie situé en Région wallonne (sud de la Belgique). Elles portent à la fois sur les moyens nécessaires à la réalisation de pareils inventaires et sur les résultats obtenus, en particulier sur la variabilité des évaluations visuelles pratiquées par différents opérateurs.

L'objet de cet article est de présenter le résultat de ces comparaisons et de préciser les conditions et les limites d'utilisation de la méthode d'inventaire par évaluation visuelle.

\section{MATÉRIEL ET MÉTHODES}

\subsection{Dispositif expérimental}

Un dispositif d'inventaire a été installé au sein d'un taillis sous futaie à réserve dense de chênes (Quercus robur L., Quercus petraea Liebl.), couvrant un peu plus de 16 ha. Ce peuplement s'apparente à une futaie claire [2] qui comporte outre les 2 chênes indigènes, des essences telles que le charme (Caprinus betulus L.), l'érable sycomore (Acer pseudoplatanus L.), le frêne (Fraxinus excelsior L.) et le merisier (Prunus avium L.). Afin de tester les diverses modalités d'inventaire à comparer, les opérations de terrain ont été menées en plusieurs

\footnotetext{
${ }^{1}$ L'acronyme UE sera utilisé ultérieurement pour unité d'échantillonnage.
}

phases durant le printemps 2002. D'abord l'inventaire complet du peuplement a été conduit par une équipe de 4 opérateurs, dont 3 mesureurs, parcourant toute la surface en virées successives.

Afin d'utiliser des centres d'UE identiques pour les différentes modalités d'inventaire par échantillonnage, les sommets d'une maille carrée de $50 \mathrm{~m}$ de côté ont été matérialisés sur le terrain. Soixante-trois UE circulaires de 10 ares, centrées sur ces sommets, ont alors été installées, fixant ainsi le taux de sondage à $40 \%$. Elles ont été parcourues en inventaire par évaluation visuelle, successivement par deux équipes de deux opérateurs puis par un opérateur seul. Deux modalités d'inventaire par évaluation visuelle ont donc été testées et la modalité à deux opérateurs a été répétée avec des personnes différentes en vue d'appréhender l'effet « opérateur » sur la variabilité des évaluations visuelles.

De plus, afin de fournir une référence pour apprécier l'impact des évaluations visuelles réalisées par différents opérateurs, deux d'entre eux ont visité 30 UE (soit 1 UE sur 2) pour y mesurer les circonférences de tous les arbres qu'elles comportaient. Le tableau I est relatif aux types et modalités d'inventaire testés.

\subsection{Récolte des données}

Lors de l'inventaire complet, toutes les tiges dépassant le seuil d'inventaire, fixé conventionnellement à $40 \mathrm{~cm}$ de circonférence à 1,5 m au-dessus du niveau du sol, ont été comptabilisées par essences et par classes de grosseur de $10 \mathrm{~cm}$ d'amplitude, sans distinction de l'origine des arbres (futaie ou taillis). Trois opérateurs ont réalisé les mesures en progressant par virées successives et en marquant d'un trait de craie les arbres mesurés dont l'essence et la classe de grosseur étaient notées par un quatrième opérateur.

L'application de la première modalité d'inventaire par évaluation visuelle a nécessité l'intervention d'une équipe de deux opérateurs dont un évaluait la grosseur des tiges en parcourant le périmètre de l'UE, de manière à pouvoir vérifier, le cas échéant, l'appartenance ou non à celle-ci des arbres situés en limite. Pour pratiquer le comptage des tiges, cet opérateur se déplace « en étoile », c'est-à-dire en réalisant un tour d'horizon complet, alternativement du centre de l'UE vers l'extérieur afin de n'oublier aucun arbre. Il se dirige vers les tiges dont il souhaite vérifier l'appartenance à une classe de grosseur ${ }^{2}$, soit en mesurant leur circonférence au ruban, soit à l'aide d' un gabarit pour les arbres proches du seuil d'inventaire. Le second opérateur se positionne au centre de l'UE, note les évaluations pratiquées et vérifie, en suivant le tour d'horizon de son équipier, que tous les arbres sont comptabilisés.

Toutes les tiges dépassant le seuil d'inventaire ont été dénombrées sans distinction de leur appartenance à la futaie ou au taillis. Elles ont été ventilées par essences et selon 5 classes de grosseur, d'une amplitude avoisinant les $50 \mathrm{~cm}$ de circonférence. Les limites précises de ces classes sont adaptées aux souhaits du gestionnaire, pour correspondre à des classes marchandes $(40-89 \mathrm{~cm}, 90-149 \mathrm{~cm}, 150-199 \mathrm{~cm}, 200$ $249 \mathrm{~cm}, 250 \mathrm{~cm}$ et plus).

\footnotetext{
${ }^{2}$ Cette vérification concerne en moyenne 5 à $10 \%$ des arbres inventoriés.
} 
Dans le cas de la seconde modalité, la procédure utilisée est similaire, si ce n'est que le seul opérateur présent sur l'UE prend en charge l'ensemble des opérations, ce qui implique un travail plus fastidieux que dans la modalité à deux opérateurs, particulièrement lorsqu'il s'avère indispensable de vérifier la grosseur de certains arbres. Il convient cependant de préciser que les deux modalités de mise en œuvre telles que décrites se révèlent spécialement efficaces en utilisant un mesureur de distance de type Vertex [9] plutôt qu'un mesureur classique (chevillère suédoise ${ }^{3}$, par exemple).

Une UE sur deux a également été visitée par une équipe de deux opérateurs procédant aux mesures, au centimètre près, des circonférences de chaque tige ayant atteint ou dépassé le seuil d'inventaire et appartenant indifféremment à la futaie ou au taillis.

Dans tous les cas, qu'il s'agisse d'inventaire complet ou d'inventaire par échantillonnage, les temps de mise en œuvre ont également été relevés.

\subsection{Traitement des données}

\subsubsection{Calcul des nombres de tiges et des surfaces terrières par ha}

Les premiers traitements effectués ont consisté à calculer, pour chaque type d'inventaire réalisé, le nombre de tiges et la surface terrière par hectare, tous deux ventilés par essences et par classes de grosseur. En ce qui concerne l'inventaire complet, le nombre de tiges par ha résulte du ratio du nombre total de tiges mesurées (éventuellement réparties par classes de grosseur ou par essences) à l'hectare de surface du peuplement inventorié. En ce qui concerne les inventaires par échantillonnage (que ce soit avec mesures ou évaluations des grosseurs), le nombre de tiges par ha (total ou par classes de grosseur) résulte des équations suivantes (Éq. (1))

$$
\begin{aligned}
& \text { Nha }=\frac{1}{m} \sum_{i=1}^{m} n_{i} \cdot \text { fext }_{i} \\
& N h a_{j}=\frac{1}{m} \sum_{i=1}^{m} n_{i j} \cdot \text { fext }_{i}
\end{aligned}
$$

avec :

$$
\text { fext }_{i}=\frac{100}{S_{i}}
$$

et sachant que :

Nha $=$ nombre total de tiges par ha ;

$\mathrm{m}=$ nombre d'UE ;

$n_{i}=$ nombre de tiges observées dans l'UE $i$;

fext $_{i}=$ facteur d'extension des UE $i$;

$N h a_{j}=$ nombre de tiges par ha pour la classe de grosseur $j$;

$n_{i j}=$ nombre de tiges de la classe de grosseur $j$ observées dans l'UE $i$;

$S_{i}=$ surface de l'UE $i$ (en ares).

Le calcul de la surface terrière dans le cas de l'inventaire complet considère la valeur centrale des classes de $10 \mathrm{~cm}$ d'amplitude comme valeur de circonférence pour chacun des arbres qui composent ces mêmes classes. La surface terrière par classe ainsi obtenue est alors ramenée à l'hectare, sur base de la surface du peuplement inventorié (Éq. (2)).

$$
\begin{gathered}
G h a=\frac{1}{4 \cdot \pi \cdot S_{t}} \sum_{j=1}^{p} \sum_{k=1}^{q_{j}} c_{k}^{2} \\
G h a_{j}=\frac{1}{4 \cdot \pi \cdot S_{t}} \sum_{k=1}^{q_{j}} c_{k}^{2}
\end{gathered}
$$

\footnotetext{
${ }^{3}$ Ruban métallique de 15 ou 25 m à enroulement automatique.
}

sachant que :

Gha $=$ surface terrière par ha (en $\left.\mathrm{m}^{2} / \mathrm{ha}\right)$;

$S_{t}=$ surface totale du peuplement inventorié (en ha);

$p=$ nombre de classes de grosseur ;

$q_{j}=$ nombre de tiges de la classe de grosseur $j$ mesurées par l'inventaire complet ;

$c_{k}=$ circonférence de l'arbre $k$ (en m);

$G h a_{j}=$ surface terrière par ha de la classe de grosseur $j\left(\mathrm{en} \mathrm{m}^{2} / \mathrm{ha}\right)$.

Connaissant la circonférence de chaque arbre mesuré dans le cas de l'inventaire par échantillonnage avec mesures des grosseurs, la surface terrière par hectare (totale ou par classes de grosseur) est calculée selon les équations suivantes (Éq. (3))

$$
\begin{gathered}
\text { Gha }=\frac{1}{4 . \pi \cdot m} \sum_{i=1}^{m} \sum_{j=1}^{p} \sum_{k=1}^{n i j} c_{i j k}^{2} \cdot \text { fext }_{i} \\
G h a_{j}=\frac{1}{4 . \pi \cdot m} \sum_{i=1}^{m} \sum_{k=1}^{n i j} c_{i j k}^{2} \cdot \text { fext }_{i},
\end{gathered}
$$$$
\text { avec : } \quad \operatorname{fext}_{i}=\frac{100}{S_{i}},
$$

et sachant que :

Gha $=$ surface terrière par ha $\left(\mathrm{en} \mathrm{m}^{2} / \mathrm{ha}\right)$;

$m=$ nombre d'UE;

$p=$ nombre de classes de grosseur ;

$n_{i j}=$ nombre de tiges de la classe de grosseur $j$ observées dans l'UE $i$;

$c_{i j k}=$ circonférence de l'arbre $k$ de la classe de grosseur $j$ mesuré dans l'UE $G h a_{j}=$ surface terrière par ha de la classe de grosseur $j$ (en $\mathrm{m}^{2} /$ ha) ;

$i$ (en $\mathrm{m})$;

fext $_{i}=$ facteur d'extension de l'UE $i$;

$S_{i}=$ surface de l'UE $i$ (en ares).

Le calcul de la surface terrière est plus problématique dans le cas des inventaires par évaluation visuelle. L'amplitude des classes de circonférence dans lesquelles sont rangés les arbres est telle que l'assimilation de la circonférence de chaque arbre à la valeur centrale de classe peut conduire à des erreurs systématiques importantes [11] C'est la raison pour laquelle il s'est avéré préalablement nécessaire de redistribuer les tiges répertoriées en classes de circonférence de $10 \mathrm{~cm}$ d'amplitude. À cet effet, un modèle d'ajustement à 6 paramètres a été retenu (Éq. (4)).

$$
N_{i}=P_{1}+\frac{P_{2}}{C_{i}{ }^{3}}+P_{4} \cdot e^{-\left(\frac{c_{i}-P_{5}}{P_{6}}\right)^{2}},
$$

avec :

$N_{i}=$ effectif estimé de la classe de grosseur $i$

$P_{1}, \ldots, P_{6}=$ paramètres de la courbe, à déterminer ;

$c_{i}=$ circonférence attribuée à la classe de circonférence $i$ (valeur centrale de la classe) s'échelonnant de $45 \mathrm{~cm}$ à la circonférence maximale par pas de $10 \mathrm{~cm}$.

Ce modèle résulte de l' addition de 2 sous-modèles (Fig. 1), l'un correspondant à une distribution typique des futaies irrégulières et l'autre à une distribution typique des futaies régulières. La somme de ces deux sous-modèles permet de prendre en compte les nombreux cas intermédiaires (Fig. 1).

Les paramètres « $\mathrm{P}_{\mathrm{i}} »$ de cette courbe sont déterminés par programmation mathématique de manière à minimiser la somme des carrés des écarts entre les nombres de tiges par classes de grosseur estimés par évaluation visuelle, d'une part, et par la sommation au sein de ces mêmes catégories des estimations obtenues par ajustement (Éq. (4)), d'autre part. L'estimation de ces paramètres s'effectue pour l'ensemble de la zone inventoriée et, le cas échéant, essence par essence. 

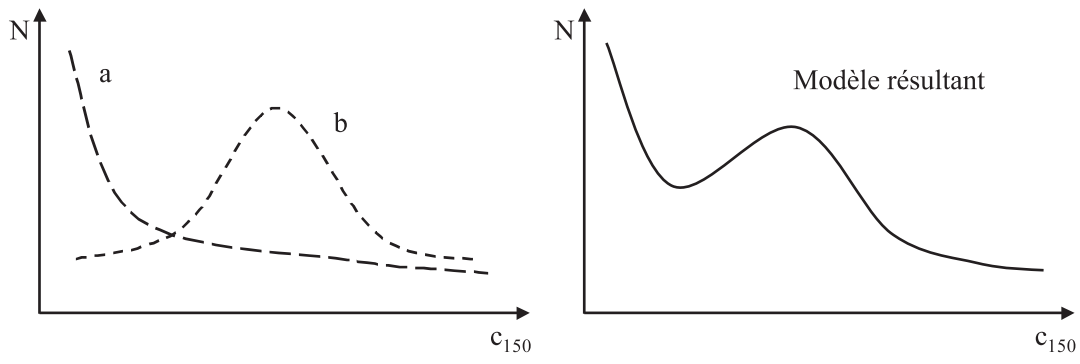

Figure 1. Construction d'un modèle de distribution des tiges $(\mathrm{N})$ par classes de circonférence (c150) résultant de la juxtaposition (a) d'un sousmodèle typique des futaies irrégulières et (b) d'un sous-modèle typique des futaies régulières.

\subsubsection{Comparaison de l'inventaire par évaluation visuelle avec l'inventaire complet}

Des tests de conformité des moyennes ont tout d'abord été effectués en considérant, d'une part, les nombres de tiges et surfaces terrières par hectare totaux obtenus en inventaire par évaluation visuelle et, d'autre part, ces mêmes variables résultant de l'inventaire complet ayant valeur de référence. Ensuite, pour apprécier la pertinence de la distribution des tiges obtenue par évaluation visuelle après sa redistribution, par ajustement, en classes de $10 \mathrm{~cm}$ d'amplitude, nous avons réalisé un test $\chi^{2}$ (Chi-carré) et calculé la valeur de l'indice de Reynolds (Éq. (5)) $[8,10]$. Cet indice permet d'apprécier la proportion de tiges classées erronément par rapport à la distribution de référence qui, dans le cas présent, est fournie par l'inventaire complet.

$$
R_{e}=\frac{\sum_{i=1}^{n} \mid N h a_{i}-\text { Nharef }_{i} \mid}{\text { Nharef }} \cdot 100
$$

avec

$N h a_{i}=$ effectif total par ha pour la classe $i$ de la distribution à tester,

Nhare $f_{i}=$ effectif total par ha pour la classe $i$ de la distribution de référence,

Nharef $=$ effectif total par ha pour la distribution de référence,

$n=$ nombre de classes de grosseur,

$R_{e}=$ indice de Reynolds exprimé en valeur relative (\%).

\subsubsection{Appréciation de l'effet « opérateur » sur les résultats obtenus}

Pour apprécier l'importance de l'effet opérateur de la méthode par évaluation visuelle, les résultats de chacune des trois modalités de cet inventaire (soit les deux répétitions de la modalité à deux opérateurs et la modalité à un opérateur) ont été confrontés à ceux obtenus en mesurant les circonférences des arbres, au sein de la moitié des UE (soit 30). Une analyse de la variance à 2 critères a été effectuée. Un premier critère fixe est constitué des 3 modalités d'inventaire par évaluation visuelle et de l'inventaire par échantillonnage avec mesures des grosseurs, ce dernier étant considéré comme témoin. Un second critère, aléatoire, est représenté par les 30 UE de 10 ares communes aux quatre modalités d'inventaire.

Cette analyse, qui a été réalisée à la fois pour le nombre de tiges et la surface terrière par ha a été complétée par un test de Dunett afin de mettre en évidence les écarts par rapport au témoin [4].

\subsubsection{Comparaison des temps de mise en ouvre et du type de résultats obtenus}

Le temps de mise en œuvre des inventaires par évaluation visuelle a été comparé à celui requis pour l'exécution de l'inventaire complet, en considérant les opérations de terrain et en faisant abstraction de la phase de bureau (préparation de l'inventaire et encodage des données).

\section{RÉSULTATS ET DISCUSSION}

\subsection{Comparaisons avec l'inventaire complet}

Les résultats, exprimés en nombres de tiges et surfaces terrières par hectare, obtenus avec les inventaires complets et par évaluation visuelle ainsi que les conclusions des tests de conformité sont présentés dans les tableaux II et III. La figure 2 présente les distributions des nombres de tiges par catégories de $10 \mathrm{~cm}$ d'amplitude, respectivement issues de l'inventaire complet et des 3 modalités de réalisation de l'inventaire par évaluation visuelle (après redistribution des tiges par ajustement). Les indices de Reynolds appliqués à ces mêmes distributions pour les 3 modalités d'évaluation sont de 11,9\% dans les modalités à 2 opérateurs et de $11,8 \%$ pour celles à 1 opérateur.

Les tests de comparaison de moyennes n'ont pas mis en évidence de différence significative entre l'inventaire complet et les différentes modalités de l'inventaire par évaluation visuelle en ce qui concerne l'estimation du nombre de tiges ou de la surface terrière. De même, les tests $\chi^{2}$ ne permettent pas de montrer de différence entre les distributions du nombre de tiges par classes de circonférence de $10 \mathrm{~cm}$ établies à partir des différentes modalités d'inventaire par évaluation visuelle et la distribution obtenue avec l'inventaire complet. Ce constat est confirmé par les valeurs très stables des indices de Reynolds, de l'ordre de $12 \%$ pour chacune des modalités.

\subsection{Appréciation de l'effet « opérateur»}

Par contre, la comparaison, sur 30 UE, des modalités de l'inventaire par évaluation visuelle avec l'inventaire par échantillonnage comportant des mesures dendrométriques fait apparaître des différences hautement significatives $(p=0,001)$ dans le cas du nombre de tiges. Le test de Dunett montre que seule la modalité à un opérateur s'écarte significativement du témoin, la sous-estimation observée restant cependant peu importante puisqu'elle atteint à peine $5 \%$ (Tab. IV). Une analyse des données de base montre que cette sous-estimation apparaît principalement dans le cas d'UE comportant un nombre important de petits bois (2 UE sur 30). On peut penser que l'opérateur travaillant seul sur le terrain est plus enclin à omettre des arbres dans de telles conditions qu'une équipe de 2 opérateurs.

L'analyse réalisée dans le cas de la surface terrière ne met pour sa part en évidence aucune différence significative entre les 3 modalités par évaluation visuelle et l'inventaire témoin (Tab. V). 
Tableau II. Nombre de tiges par hectare $\left(\mathrm{ha}^{-1}\right)$ : valeurs totales et valeurs par classes de grosseur obtenues avec les différents inventaires. Les valeurs entre parenthèses correspondent aux différences (en \%) entre les valeurs estimées par évaluation visuelle et les valeurs de référence (inventaire complet). Tests de comparaison de moyennes (hypothèse nulle = égalité des valeurs totales entre les modalités " évaluation visuelle » et l'inventaire complet, pour $\alpha=5 \%$ ). Test $\chi^{2}$ (hypothèse nulle = équivalence entre les distributions des modalités «Inventaire visuel » et celle de l'inventaire complet, pour $\alpha=5 \%$ ) et indice de Reynolds (Re en $\%$ ) relatifs à la distribution des tiges après redistribution en classes de $10 \mathrm{~cm}$ d'amplitude.

\begin{tabular}{|c|c|c|c|c|c|c|c|c|c|}
\hline \multirow[t]{2}{*}{ NHA } & \multicolumn{5}{|c|}{ Classes de grosseur } & \multirow[t]{2}{*}{ Total } & \multirow[t]{2}{*}{ Comparaison de moyennes } & \multirow[t]{2}{*}{ Test $\chi^{2}$} & \multirow[t]{2}{*}{$\operatorname{Re}(\%)$} \\
\hline & $40-90$ & $90-150$ & $150-200$ & $200-250$ & $250-300$ & & & & \\
\hline Inventaire complet & 177,3 & 52,3 & 17,5 & 9,8 & 7,0 & 263,9 & Rejet de l'hypothèse nulle & Rejet de l'hypothèse nulle & \\
\hline \multicolumn{10}{|l|}{$\begin{array}{l}\text { Inventaire « évaluation } \\
\text { visuelle» }\end{array}$} \\
\hline Modalité 2 opérateurs a ${ }^{a}$ & $\begin{array}{l}186,2 \\
(+5,0)\end{array}$ & $\begin{array}{c}52,5 \\
(+0,4)\end{array}$ & $\begin{array}{c}18,1 \\
(+3,4)\end{array}$ & $\begin{array}{c}13,5 \\
(+37,8)\end{array}$ & $\begin{array}{c}4,8 \\
(-31,4)\end{array}$ & $\begin{array}{l}277,8 \\
(+5,3)\end{array}$ & $\begin{array}{c}\text { non } \\
(p=0.409)\end{array}$ & $\begin{array}{c}\text { non } \\
(p=0,005)\end{array}$ & 11,9 \\
\hline Modalité 2 opérateurs b & $\begin{array}{l}188,3 \\
(+6,2)\end{array}$ & $\begin{array}{c}52,4 \\
(+0,2)\end{array}$ & $\begin{array}{c}18,3 \\
(+4,6)\end{array}$ & $\begin{array}{c}13,3 \\
(+35,7)\end{array}$ & $\begin{array}{c}5,6 \\
(-20,0)\end{array}$ & $\begin{array}{l}275,1 \\
(+4,2)\end{array}$ & $\begin{array}{c}\text { non } \\
(p=0.582)\end{array}$ & $\begin{array}{c}\text { non } \\
(p=0,006)\end{array}$ & 11,9 \\
\hline Modalité 1 opérateur & $\begin{array}{l}175,1 \\
(-1,2)\end{array}$ & $\begin{array}{c}53,8 \\
(+2,9)\end{array}$ & $\begin{array}{c}18,1 \\
(+3,4)\end{array}$ & $\begin{array}{c}13,7 \\
(+39,8)\end{array}$ & $\begin{array}{c}4,6 \\
(-34,3)\end{array}$ & $\begin{array}{l}265,2 \\
(+0,5)\end{array}$ & $\begin{array}{c}\text { non } \\
(p=0.609)\end{array}$ & $\begin{array}{c}\text { non } \\
(p=0,003)\end{array}$ & 11,8 \\
\hline
\end{tabular}

a Les deux répétitions de l'inventaire par évaluation visuelle à 2 opérateurs seront caractérisées par «modalité 2 opérateurs a » et «modalité 2 opérateurs $\mathrm{b} \gg$ dans la suite de cet article.

Tableau III. Surface terrière par hectare $\left(\mathrm{m}^{2} \cdot \mathrm{ha}^{-1}\right)$ : valeurs totales et valeurs par classes de grosseur obtenues avec les différents inventaires. Les valeurs entre parenthèses correspondent aux différences (en \%) entre les valeurs estimées par évaluation visuelle et les valeurs de référence (inventaire complet). Tests de comparaison de moyennes (hypothèse nulle = égalité des valeurs totales entre les modalités " évaluation visuelle » et l'inventaire complet, pour $\alpha=5 \%$ ).

\begin{tabular}{|c|c|c|c|c|c|c|c|}
\hline \multirow[b]{2}{*}{ GHA } & \multicolumn{5}{|c|}{ Classes de grosseur } & \multirow[t]{2}{*}{ Total } & \multirow[t]{2}{*}{ Comparaison de moyennes } \\
\hline & $40-90$ & $90-150$ & $150-200$ & $200-250$ & $250-300$ & & \\
\hline Inventaire complet & 5,11 & 5,65 & 4,25 & 3,76 & 4,21 & 22,98 & Rejet de l'hypothèse nulle \\
\hline \multicolumn{8}{|c|}{ Inventaire « évaluation visuelle » } \\
\hline Modalité 2 opérateurs a & $\begin{array}{c}5,26 \\
(+2,9)\end{array}$ & $\begin{array}{c}5,61 \\
(-0,7)\end{array}$ & $\begin{array}{c}4,32 \\
(+1,6)\end{array}$ & $\begin{array}{c}5,37 \\
(+42,8)\end{array}$ & $\begin{array}{c}2,94 \\
(-30,2)\end{array}$ & $\begin{array}{l}23,50 \\
(+2,3)\end{array}$ & $\begin{array}{c}\text { non } \\
(p=0,430)\end{array}$ \\
\hline Modalité 2 opérateurs b & $\begin{array}{c}5,34 \\
(+4,5)\end{array}$ & $\begin{array}{c}5,60 \\
(-0,9)\end{array}$ & $\begin{array}{c}4,35 \\
(+2,4)\end{array}$ & $\begin{array}{c}5,30 \\
(+41,0)\end{array}$ & $\begin{array}{c}3,42 \\
(-18,8)\end{array}$ & $\begin{array}{l}24,01 \\
(+4,5)\end{array}$ & $\begin{array}{c}\text { non } \\
(p=0,164)\end{array}$ \\
\hline Modalité 1 opérateur & $\begin{array}{c}4,95 \\
(-3,1)\end{array}$ & $\begin{array}{c}5,75 \\
(+1,8)\end{array}$ & $\begin{array}{c}4,32 \\
(+1,6)\end{array}$ & $\begin{array}{c}5,43 \\
(+44,4)\end{array}$ & $\begin{array}{c}2,84 \\
(-32,5)\end{array}$ & $\begin{array}{l}23,29 \\
(+1,3)\end{array}$ & $\begin{array}{c}\text { non } \\
(p=0,636)\end{array}$ \\
\hline
\end{tabular}

\subsection{Temps de réalisation et utilisation des résultats}

Les vitesses d'exécution des différents inventaires prennent en considération les temps de mesure au sein des UE ainsi que les temps de cheminement et ceux d'installation de celles-ci. Les temps d'encodage des données et de traitement des résultats étant considérés comme équivalents, ils n'ont pas été inclus dans la comparaison. Les temps d'exécution liés aux inventaires par échantillonnage sont quelque peu sous-estimés dans la mesure où les contraintes expérimentales ont conduit à matérialiser au préalable les centres d'UE, alors qu'en condition réelle les cheminements entre UE doivent aussi inclure la recherche du centre de l'UE suivante (mesure de distance et visée à la boussole forestière).

L'inventaire complet qui s'appuie sur 4 opérateurs permet de parcourir $3,3 \mathrm{ha} / \mathrm{ho} / \mathrm{j}^{4}$ pour $4,4 \mathrm{ha} / \mathrm{ho} / \mathrm{j}$ dans le cas de l'inven-

\footnotetext{
${ }^{4}$ ha/ho/j: hectare par homme par jour.
}

taire par échantillonnage ( 2 opérateurs) tandis que les modalités d'inventaire visuel à 2 opérateurs et à 1 seul opérateur permettent de couvrir respectivement 5,2 ha/ho/j et 9,5 ha/ho/j.

On constate que la vitesse d'exécution de la modalité d'inventaire par évaluation visuelle avec 2 opérateurs est proche de celle de l'inventaire par échantillonnage avec mesures des grosseurs, elle-même plus élevée que la vitesse d'exécution de l'inventaire complet. L'inventaire par évaluation visuelle à 1 opérateur présente une efficacité accrue avec une vitesse de réalisation de l'ordre de 2 fois supérieure à celle de la modalité à 2 opérateurs et 3 fois supérieure à celle de l'inventaire complet.

Les vitesses d'exécution des inventaires par échantillonnage sont calculées dans l'hypothèse de l'utilisation d'un dendromètre à ultrasons. Ce type d'appareil bien que relativement coûteux $(\sim 1500 €)^{5}$ peut être très vite amorti dès lors que son

\footnotetext{
${ }^{5}$ Prix TTC en 2004.
} 


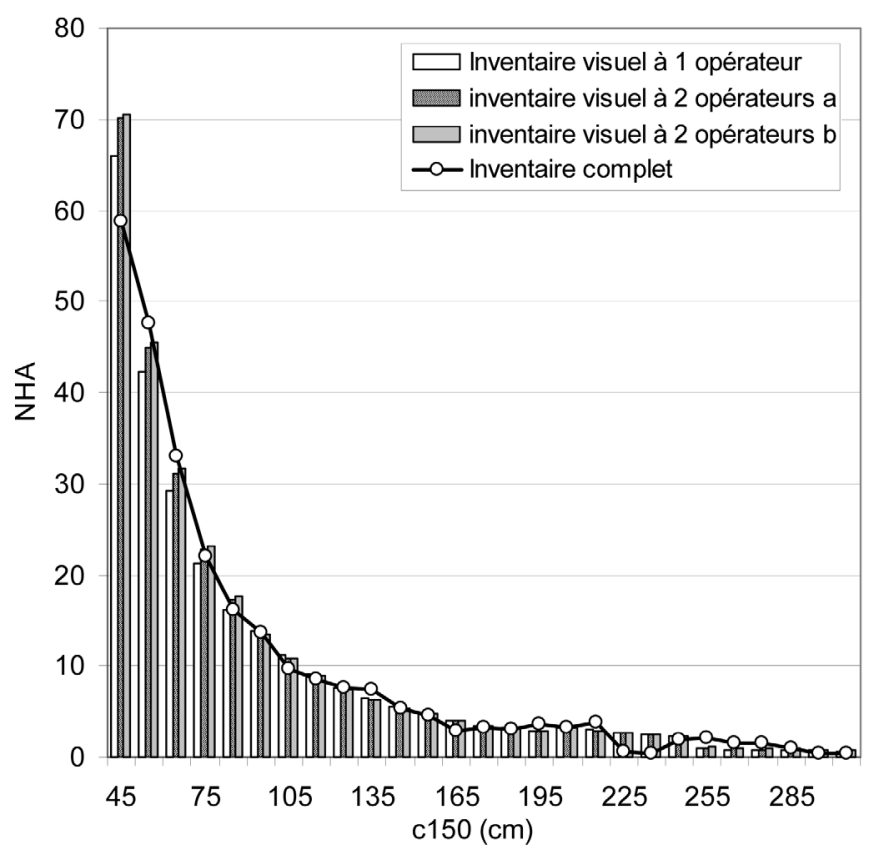

Figure 2. Distributions du nombre de tiges par hectare (NHA) par classes de circonférence (c150) pour l'inventaire complet et pour les différentes modalités d'inventaire par évaluation visuelle.

utilisation est fréquente et que les surfaces inventoriées sont importantes.

Alors que l'inventaire par évaluation visuelle s'avère être plus intéressant que l'inventaire complet du point de vue de sa vitesse d'exécution, il fournit, de surcroît, une représentation spatiale des résultats. Seuls les aspects dendrométriques ont été abordés dans cette étude, mais la méthode proposée est très souple, et sans alourdir pour autant les opérations de terrain, elle permet également de récolter des données relatives à la régénération, au taillis, ou encore à l'état sanitaire, autant d'infor- mations revêtant une très grande utilité pour le gestionnaire lors de la réalisation de la phase d'analyse des aménagements [5].

\section{CONCLUSIONS}

La méthode d'inventaire par évaluation visuelle telle que proposée peut être considérée comme une alternative recommandable à l'inventaire complet, aussi bien en termes de précision des résultats que de vitesse d'exécution. Parmi les tests réalisés, seule la comparaison entre la modalité à un opérateur et un inventaire avec mesures des grosseurs (considéré comme témoin) a mis en évidence une très légère sous-estimation du nombre de tiges par ha.

La méthode par évaluation visuelle permet en outre d'obtenir une information plus riche que l'inventaire complet, en donnant la possibilité de spatialiser les résultats (cartographie), en mobilisant des moyens humains au moins deux fois plus réduits pour une même précision.

De plus, la possibilité de recourir à un ajustement permettant de redistribuer les nombres de tiges par hectare en classes de grosseur d'amplitude plus faible, permet l'allègement des protocoles de mesure et facilite les opérations de terrain par comparaison avec la mise en œuvre d'un inventaire par échantillonnage où tous les bois sont mesurés.

La modalité d'inventaire par évaluation visuelle à un opérateur présente les résultats les plus intéressants en termes de vitesse d'exécution : elle est en effet deux fois plus rapide à mettre en œuvre que la variante à deux opérateurs et trois fois plus rapide que l'inventaire complet. Il convient cependant de nuancer ces conclusions en insistant sur le caractère relativement fastidieux de ce type d'inventaire lorsqu'il est pratiqué par un seul opérateur, surtout en présence d'un nombre important de petits bois, avec un risque d'oubli dans le dénombrement de ces petits bois. Rappelons en outre que les résultats enregistrés, au plan de la vitesse d'exécution, supposent l'utilisation d'un dendromètre à ultrasons pour les mesures de distance.

Tableau IV. Nombre de tiges par hectare : valeurs totales et valeurs par classes de grosseurs obtenues au départ des 30 UE visitées à la fois en inventaire par évaluation visuelle et en inventaire par échantillonnage avec mesures des grosseurs. Les valeurs entre parenthèses correspondent aux différences (en \%) entre les valeurs estimées par évaluation visuelle et les valeurs de référence (inventaire avec mesures). Analyse de la variance : niveau de signification de l'effet opérateur $(\alpha=5 \%)$. Test de Dunett : niveau de signification de la différence entre les modalités de l'inventaire par évaluation visuelle et le témoin $(\alpha=5 \%)$.

\begin{tabular}{|c|c|c|c|c|c|c|c|c|}
\hline \multirow[t]{2}{*}{ NHA } & \multicolumn{5}{|c|}{ Classes de grosseur } & & \multirow{2}{*}{$\begin{array}{c}\text { Signification } \\
\text { de l'effet « opérateur » }\end{array}$} & \multirow[t]{2}{*}{ Test de Dunett } \\
\hline & $40-90$ & $90-150$ & $150-200$ & $200-250$ & $250-300$ & Total & & \\
\hline $\begin{array}{l}\text { Inventaire par échantillonnage } \\
\text { avec mesures des grosseurs }\end{array}$ & 175,0 & 56,7 & 18,3 & 16,0 & 4,3 & 266,0 & & \\
\hline Inventaire « évaluation visuelle» & & & & & & & $p=0,066$ & \\
\hline Modalité 2 opérateurs a & $\begin{array}{l}172,7 \\
(-1,3)\end{array}$ & $\begin{array}{c}58,3 \\
(+2,8)\end{array}$ & $\begin{array}{c}19,0 \\
(+3,8)\end{array}$ & $\begin{array}{c}14,7 \\
(-8,1)\end{array}$ & $\begin{array}{c}4,7 \\
(+9,3)\end{array}$ & $\begin{array}{l}264,7 \\
(-0,5)\end{array}$ & & $p=0,991$ \\
\hline Modalité 2 opérateurs b & $\begin{array}{l}176,3 \\
(+0,7)\end{array}$ & $\begin{array}{c}58,7 \\
(+3,5)\end{array}$ & $\begin{array}{c}18,0 \\
(-1,6)\end{array}$ & $\begin{array}{l}15,0 \\
(-6,3)\end{array}$ & $\begin{array}{c}5,7 \\
(+32,6)\end{array}$ & $\begin{array}{l}268,0 \\
(+0,8)\end{array}$ & & $p=0,763$ \\
\hline Modalité 1 opérateur & $\begin{array}{l}161,3 \\
(-7,8)\end{array}$ & $\begin{array}{c}58,3 \\
(+2,8)\end{array}$ & $\begin{array}{c}18,0 \\
(-1,6)\end{array}$ & $\begin{array}{c}15,0 \\
(-6,3)\end{array}$ & $\begin{array}{c}4,0 \\
(-7,0)\end{array}$ & $\begin{array}{l}252,7 \\
(-5,0)\end{array}$ & & $p=0,043$ \\
\hline
\end{tabular}


Tableau V. Surface terrière par hectare $\left(\mathrm{m}^{2} \cdot \mathrm{ha}^{-1}\right)$ : valeurs totales et valeurs par classes de grosseurs obtenues au départ des 30 UE visitées à la fois en inventaire par évaluation visuelle et en inventaire par échantillonnage avec mesures des grosseurs Les valeurs entre parenthèses correspondent aux différences (en \%) entre les valeurs estimées par évaluation visuelle et les valeurs de référence (inventaire avec mesures). Analyse de la variance : niveau de signification de l'effet opérateur $(\alpha=5 \%)$. Test de Dunett : niveau de signification de la différence entre les modalités de l'inventaire par évaluation visuelle et le témoin $(\alpha=5 \%)$.

\begin{tabular}{|c|c|c|c|c|c|c|c|c|}
\hline \multirow[t]{2}{*}{ GHA } & \multicolumn{5}{|c|}{ Classes de grosseur } & & \multirow{2}{*}{$\begin{array}{c}\text { Signification de } \\
\text { l'effet « opérateur » }\end{array}$} & \multirow[t]{2}{*}{ Test de Dunett } \\
\hline & $40-90$ & $90-150$ & $150-200$ & $200-250$ & $250-300$ & Total & & \\
\hline $\begin{array}{l}\text { Inventaire par échantillonnage } \\
\text { avec mesures des grosseurs }\end{array}$ & 4,7 & 6,2 & 4,5 & 6,1 & 2,5 & 23,9 & & \\
\hline Inventaire « évaluation visuelle & & & & & & & $p=0,001$ & \\
\hline Modalité 2 opérateurs a & $\begin{array}{c}4,9 \\
(+4,3)\end{array}$ & $\begin{array}{c}6,2 \\
(0,0)\end{array}$ & $\begin{array}{c}4,5 \\
(0,0)\end{array}$ & $\begin{array}{c}5,8 \\
(-4,9)\end{array}$ & $\begin{array}{c}2,9 \\
(+16,0)\end{array}$ & $\begin{array}{c}24,4 \\
(+2,1)\end{array}$ & & $p=0,794$ \\
\hline Modalité 2 opérateurs b & $\begin{array}{c}5,0 \\
(+6,4)\end{array}$ & $\begin{array}{c}6,3 \\
(+1,6)\end{array}$ & $\begin{array}{c}4,3 \\
(-4,4)\end{array}$ & $\begin{array}{c}6,0 \\
(-1,6)\end{array}$ & $\begin{array}{c}3,5 \\
(+40,0)\end{array}$ & $\begin{array}{c}25,0 \\
(+4,6)\end{array}$ & & $p=0,152$ \\
\hline Modalité 1 opérateur & $\begin{array}{c}4,6 \\
(-2,1)\end{array}$ & $\begin{array}{c}6,2 \\
(0,0)\end{array}$ & $\begin{array}{c}4,3 \\
(-4,4)\end{array}$ & $\begin{array}{c}6,0 \\
(-1,6)\end{array}$ & $\begin{array}{c}2,5 \\
(0,0)\end{array}$ & $\begin{array}{c}23,5 \\
(-1,7)\end{array}$ & & $p=0,817$ \\
\hline
\end{tabular}

Cette réserve est cependant toute relative car ce type d'équipement, quoique coûteux, peut être amorti très rapidement dès lors que les inventaires complets sont remplacés par des inventaires par évaluation visuelle. En effet, à partir de quelques centaines d'hectares, il devient plus coûteux de pratiquer l'inventaire complet que d'investir dans un tel appareil. Enfin, le protocole de récolte de données testé dans le cadre de cette étude ne prenait en compte que les données relatives aux grosseurs des tiges. La saisie de données complémentaires, par exemple relatives à la régénération, voire à d'autres paramètres écologiques ou sylvicoles qui s'y prêteraient, pourrait conduire à considérer l'inventaire par évaluation visuelle comme un outil performant dans la phase cruciale de description d'un massif forestier. Des tests complémentaires ont été réalisés dans 2 faciès forestiers autres que la chênaie : il s'agit (i) de peuplements de feuillus nobles à composition et structure très hétérogènes et (ii) de hêtraies dont la structure est globalement équienne. Toutes ces expériences tendent à confirmer l'intérêt de la méthode.

À l'instar de toutes les autres méthodes d'inventaire existantes, celle basée sur une estimation visuelle mérite bien évidemment, comme souligné sur un plan général par Rondeux et al. [12], d'être appliquée en connaissance de cause et en fonction même des objectifs qu'elle peut rencontrer.

Remerciements: La présente étude a été réalisée avec le financement de la Région wallonne (Direction Générale des Ressources Naturelles et de l'Environnement). Elle relève plus spécialement de l'action de recherche 1.3.2 «Développement de techniques d'inventaire appliquées aux différentes facettes de la gestion forestière intégrée » de l'Accord-Cadre de Recherche forestière.

\section{REFERENCES}

[1] Aubry S., Bruciamacchie M., Druelle P., L'inventaire typologique : un outil performant pour l'élaboration des aménagements ou plans simples de gestion, Rev. For. Fr., XLII (1990) 429-444.

[2] Boudru M., Forêt et Sylviculture, Traitement des forêts, Les Presses agronomiques de Gembloux, Gembloux, 1989.

[3] Bousson E., Lejeune P., Rondeux J., L'inventaire par évaluation visuelle, une nouvelle méthode adaptée à la description de peuplements hétérogènes, Forêt Wallonne 58, Cahier technique (2002) 6-12.

[4] Dagnelie P., Théorie et méthodes statistiques. Applications agronomiques. Tome 2, Les Presses Agronomiques de Gembloux, Gembloux, 1979.

[5] Dubourdieu J., Manuel d'aménagement forestier, Office National des Forêt, Techniques et Documentation, Lavoisier, Paris, 1997.

[6] Duplat P., Perrotte G., Inventaire et estimation de l'accroissement des peuplements forestiers, Office National des Forêts, Section Technique, Fontainebleau, 1981.

[7] Hebert J., Bourland N., Rondeux J. Estimation de l'accroissement et de la production forestière à l'aide de placettes permanentes concentriques, Ann. Sci. For. 62 (2005) 229-236.

[8] Lejeune P., Construction d'un modèle de répartition des arbres par classes de grosseur pour des plantations d'épicéa commun (Picea abies (L.) Karst) en Ardenne belge, Ann. Sci. For. 51 (1994) 53-65.

[9] Pauwels D., Rondeux J., Le Forestor Vertex : une nouvelle génération de dendromètres, Rev. For. Fr. 49 (1997) 59-63.

[10] Reynolds M.R., Burk T.E., Huang W.C., Goodness-of-fit tests and model selection procedures for diameter distributions models, Forest Sci. 34 (1988) 373-399.

[11] Rondeux J., La mesure des arbres et des peuplements forestiers, Les Presses Agronomiques de Gembloux, Gembloux, 1999.

[12] Rondeux J., Hebert J., Marchal D., Les inventaires forestier de gestion : quels objectifs et quelles méthodes? J. For. Suisse 149 (1998) 463-473. 